\title{
Short- and Medium-Term Impact of a Structured Medical Intervention in Adolescents with Overweight, Obesity, or Increased Waist Circumference
}

\author{
Luisa Macieira ${ }^{a, b}$ Jorge Saraiva ${ }^{a, c, d}$ Lélita da Conceição dos Santos ${ }^{e, f}$ \\ aUniversity of Coimbra, Faculty of Medicine, University Pediatric Clinic, Coimbra, Portugal; bSuperior School of \\ Health Technology, Dietetics and Nutrition, Coimbra, Portugal; ' ${ }^{C H o s p i t a l}$ and University Centre of Coimbra, Medical \\ Genetics Unit, Coimbra, Portugal; ${ }^{d}$ Clinical Academic Centre of Coimbra, Coimbra, Portugal; eUniversity of Coimbra, \\ Faculty of Medicine, Coimbra, Portugal; ${ }^{f}$ Hospital and University Centre of Coimbra, Internal Medicine Senior \\ Graduate Hospital Assistant, Coimbra, Portugal
}

\section{Keywords}

Adolescents · Obesity · Overweight · Diet · Exercise

\begin{abstract}
Introduction and Objectives: This study aimed to assess the impact of a structured medical intervention in adolescents with overweight or obesity at medical discharge and after 5 years and identify the factors responsible for the reappearance of anthropometric alterations. Methods: A total of 42 adolescents with overweight, obesity, and/or increased waist circumference (WC) participated in a survey on eating habits, physical exercise, and sedentary habits. Body mass index (BMI), fat mass (FM), and WC were evaluated quarterly during a structured medical intervention, with a maximum duration of 3 years (2007-2010) and 5 years after its conclusion (2015-2016). SPSS $19.0^{\circledR}$ was used for statistical analysis. Results: Initially, $23.8 \%$ adolescents were overweight, 28.6\% were obese, $83.3 \%$ had increased FM, and $95.2 \%$ had high WC. A significant improvement was noted until medical discharge with respect to BMI, FM, and WC $(p<0.001)$ owing to an increased number of meals; reduction in the consumption of hypercaloric foods/drinks ( $p<0.001)$; increase in the
\end{abstract}

karger@karger.com www.karger.com/ofa

Karger $\stackrel{\text { ' }}{5}$

GOPEN ACCESS
(C) 2021 The Author(s)

Published by S. Karger AG, Basel

This is an Open Access article licensed under the Creative Commons Attribution-NonCommercial-4.0 International License (CC BY-NC) (http://www.karger.com/Services/OpenAccessLicense), applicable to the online version of the article only. Usage and distribution for commercial purposes requires written permission. consumption of fruits, vegetables, and soup $(p<0.001)$; increase in physical exercise and daily walks ( $p<0.001)$; and reduction in sedentary habits $(p<0.001)$. At reassessment, after 5 years, the majority had normal BMl, FM, and WC ( $p<$ $0.001)$, although $45.2 \%$ had abandoned sports $(p<0.001)$. Conclusions: A relation exists between the improvement or normalization of BMI, FM, and WC and the number of meals, healthy eating habits, physical exercise, and less sedentary habits. The intervention was associated with an improvement in the parameters during the short and medium terms.

(c) 2021 The Author(s)

Published by S. Karger AG, Basel

\section{Introduction}

Obesity occurs owing to the complex interaction between environmental and genetic factors [1] and was declared the 21st-century pandemic by the World Health Organization (WHO) [2]. Pediatric obesity has reached epidemic levels in developed countries and is currently the most prevalent nutritional pediatric disease [3]. An international study showed that Portugal has some of the 
most overweight adolescents [4]. Excessive fat mass (FM) results mainly from hypercaloric diets, sedentary habits, and reduced physical exercise [5].

The risks associated with obesity are related to body mass index (BMI), FM, and metabolic alterations [6]. Obese or overweight children or adolescents have higher cardiovascular and metabolic risks [7]. Furthermore, when a child becomes an obese adolescent, he/she has a high probability of becoming an obese adult [8].

The Western lifestyle encourages reduced energy expenditure by promoting a hypercaloric diet at the expense of vegetables, salads, and fruits. Excessive television viewing is related to an increased consumption of highly energetic foods and physical inactivity [9]. Exercise is essential for the prevention/treatment of obesity [10], which can counteract the effect of some genetic polymorphisms associated with juvenile obesity [11], and is considered the best nonpharmacological treatment [12] along with spending less time on sedentary habits (such as watching television and playing video games) [13].

Hypercaloric/unbalanced diet is another factor contributing to childhood overweight/obesity [14]. Changing eating habits (increasing soup, vegetable, and fruit consumption) is important in the treatment and/or preventing obesity [15].

There is an urgent need for preventive/treatment measures against obesity/overweight, particularly among adolescents. We believe that by promoting exercise, consuming healthy diet, and continuous monitoring, the current situation can be improved. These measures should be implemented from an early age as a cooperative project among schools, government, family, and specialists [16, 17], whose intervention is fundamental, particularly the family [18-21]. We aimed to assess the impact of a structured medical intervention lasting up to 3 years in overweight/obese adolescents in 2 stages (at the time of their discharge and after 5 years) and to identify the factors responsible for the aggravation of anthropometric parameters during this period.

\section{Patients and Methods}

A total of 42 participants were included in a structured medical intervention program from a group of ninety 10 - to 12 -yearold adolescents of both sexes from 2 different areas in Portugal (rural and urban). All participants were studying in a local public school (rural area) or a private school (urban area), and all had overweight/obesity and/or increased waist circumference (WC) [22]. Moreover, 48 adolescents were excluded because they were subjected to chronic pharmacological therapy, which could interfere with the risk of obesity/overweight and second- ary obesity, or because they were not authorized by their guardians.

All adolescents completed 2 surveys at the initial consultation, during quarterly assessments (up to 3 years), and in reassessment (after 5 years). Both surveys composed of closed option questions. One survey evaluated the number of meals and their menu (analyzing their eating habits), and another survey assessed out-ofschool physical activity(ies). The first survey inquired about the number of meals per day, their menu, frequency of intake of certain foods (fruits, salads/vegetables, soup, water, soft drinks, sweets, fast food, and chocolate milk) per day and per week, allowing the adolescents to choose between "never" (per day or per week) and ">4 times" (per day or week). The second survey assessed the level of sedentary activities (such as watching television or playing video games) (how many hours per day and per week) and the level, duration, and type of exercise practiced by the adolescents (football, swimming, and daily walks) (allowing the adolescents to choose between "none" and "> 4 h per week").

The number of hours was categorized into 3 groups ( $h=$ hours): $<3 \mathrm{~h} /$ week, $3-4 \mathrm{~h} /$ week, and $>4 \mathrm{~h} /$ week. The $<3 \mathrm{~h} /$ week group was associated with lower energy expenditure and $>4 \mathrm{~h} /$ week group with higher energy expenditure. The presence/absence of daily walks (>30 $\mathrm{min}$ ) was assessed, and the number of hours spent on sedentary activities (television, computer, and video games) was determined. At every consultation, weight, WC, and BMI were recorded.

The adolescents' nutritional statuses were evaluated by considering BMI as the determinant of overweight (weight $>$ P85) and obesity (weight $>$ P97), adopting the percentile curves for BMI of the $\mathrm{WHO}$ and using internationally recommended techniques and methodologies [23]. According to the WHO, for reassessment, BMI $\geq 25 \mathrm{~kg} / \mathrm{m}^{2}$ indicated overweight and BMI $\geq 30 \mathrm{~kg} / \mathrm{m}^{2}$ indicated obesity. WC was evaluated considering the midpoint between the last costal arch and iliac crest, and the values found were interpreted in the respective percentile curves [24], with body fat being determined based on bioimpedance (Tanita TB 300).

The adolescents were followed up in consultations at 3-month intervals until normalization of the altered parameters; next, they were discharged (after a maximum of 3 years). Furthermore, families and adolescents were made aware of the necessity to change their habits to normalize their weight and FM. In the rural area, the local government was involved to facilitate out-of-school sports activities for all adolescents. Exercise was encouraged during the follow-up to increase energy expenditure. The adolescents were encouraged to practice out-of-school exercise for at least $4 \mathrm{~h}$. Daily walks (<30 min) were instituted. Healthy eating and abolition/reduction of caloric foods (sweets, sugary drinks, and fast food) were promoted. Daily consumption of soup, vegetables, and salads was encouraged, and a minimum consumption of 3 pieces of fruits a day was recommended. An increase in the number of meals while sharing with the family was promoted. At 5 years after discharge, the participants (presently aged 17-21 years) were reassessed and completed the same surveys again. Next, they were subjected to nutritional status assessment with clinical assessment of the initially evaluated parameters.

For statistical analysis, the categorical variables were characterized using absolute and relative frequencies. The comparison among the initial consultation, consultation at discharge, and reassessment was concluded in relation to the variables of the food and occupational surveys and BMI, FM, and WC. The nonparametric
Intervention in Adolescents with

Overweight, Obesity, or Increased WC
Obes Facts 2021;14:622-632

DOI: $10.1159 / 000519270$ 
Table 1. Assessment of habits in the initial consultation, at discharge, and at reassessment

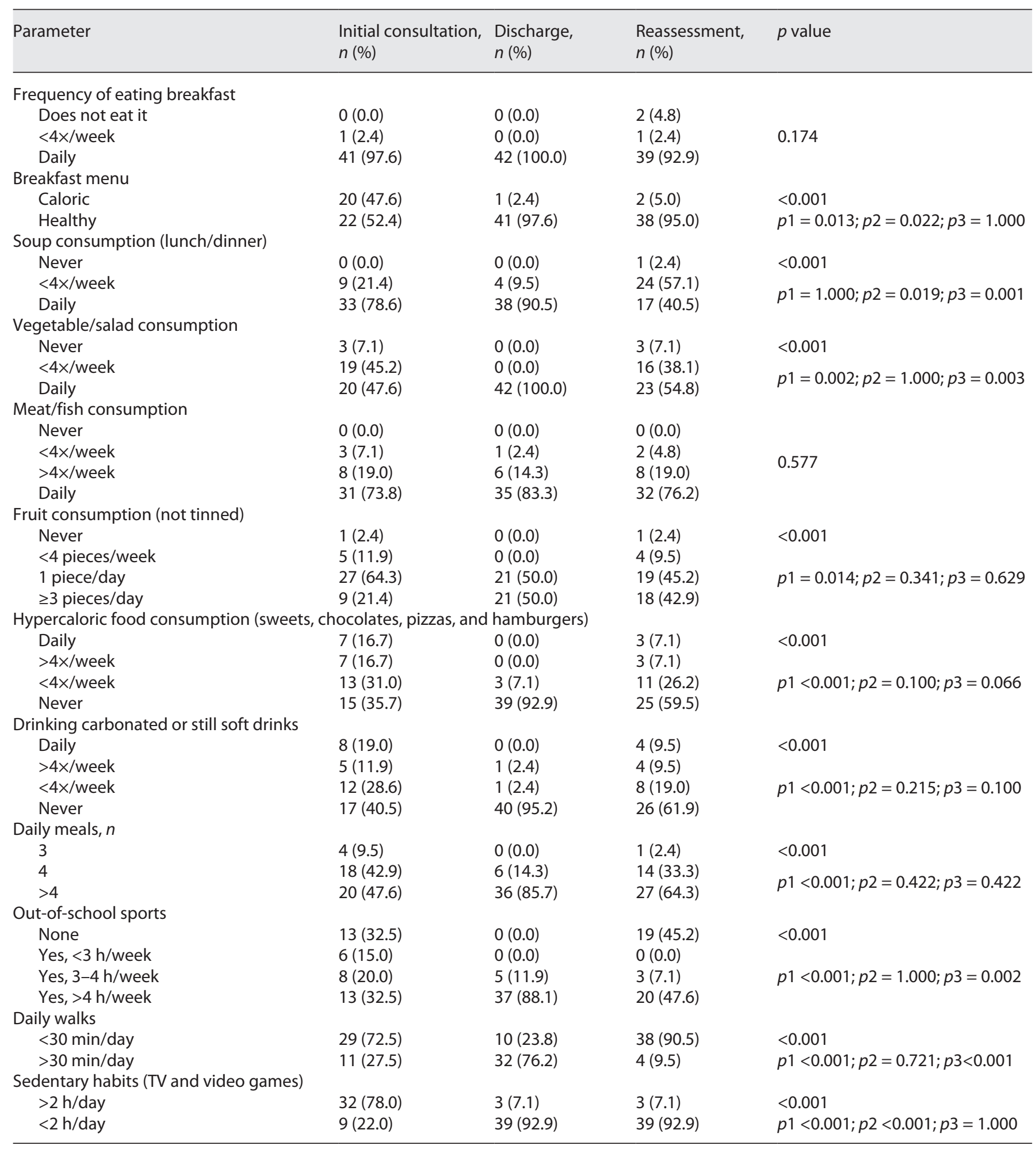

Comparison between the 3 interval measurements carried out by using Cochran's Q nonparametric (binary variables) or Friedman's (categorical variables) tests for both paired samples. Multiple comparisons: $p 1$, initial consultation versus discharge; $p 2$, initial consultation versus reassessment; $p 3$, discharge versus reassessment. 
Table 2. BMI, FM, and WC at the initial consultation, discharge, and reassessment

\begin{tabular}{lllll}
\hline Parameter & $\begin{array}{l}\text { Initial consultation, } \\
n(\%)\end{array}$ & $\begin{array}{l}\text { Discharge, } \\
n(\%)\end{array}$ & $\begin{array}{l}\text { Reassessment, } \\
n(\%)\end{array}$ & $p$ value \\
\hline BMI & & & \\
$\quad$ Normal & $20(47.6)$ & $42(100.0)$ & $35(83.3)$ & $<0.001$ \\
$\quad \begin{array}{l}\text { Overweight } \\
\text { Obesity }\end{array}$ & $10(23.8)$ & $0(0.0)$ & $6(14.3)$ & $p 1=0.001 ; p 2=0.023 ; p 3=0.900$ \\
FM & $12(28.6)$ & $0(0.0)$ & $1(2.4)$ & \\
$\quad$ Normal & $7(16.7)$ & $42(100.0)$ & $31(73.8)$ & $<0.001$ \\
$\quad$ High & $16(38.1)$ & $0(0.0)$ & $9(21.4)$ & $p 1<0.001 ; p 2<0.001 ; p 3=0.305$ \\
$\quad$ Very high & $19(45.2)$ & $0(0.0)$ & $2(4.8)$ & $<0.001$ \\
WC & $2(4.8)$ & $40(95.2)$ & $35(83.3)$ & $p 1<0.001 ; p 2<0.001 ; p 3=1.000$ \\
$\quad \begin{array}{l}\text { Normal } \\
\text { Increased }\end{array}$ & $14(33.3)$ & $2(4.8)$ & $5(11.9)$ & $2(4.8)$ \\
$\quad$ Obesity & $26(61.9)$ & $0(0.0)$ & & \\
\hline
\end{tabular}

Comparison between the interval measurements carried out by using the nonparametric Friedman's test for paired samples. Multiple comparisons: $p 1$, initial consultation versus discharge; $p 2$, initial consultation versus reassessment; $p 3$, discharge versus reassessment. BMI, body mass index; FM, fat mass; WC, waist circumference.

tests for paired samples by Friedman and Cochran's Q were used for comparison in relation to categorical variables and binary variables, respectively.

For each end point (worsening of BMI, FM, and WC) between discharge and reassessment, the associations were tested with the following variables using univariable logistic regression models: gender, environment, education, age, and changes in eating and occupational behavior. Multivariate logistic regression models were subsequently adjusted to identify independent predictors. The Wald test was used to assert whether the impact of the variable on the occurrence of the end point is significant. The variables in the multivariable models were selected using the stepwise forward method with a likelihood ratio test. Moreover, the adjusted odds ratio and respective 95\% confidence intervals were estimated.

A significance level was considered at 5\%. Statistical analysis was performed using SPSS $19.0^{\circledR}$.

\section{Results}

Of the 42 adolescents who participated in the study, $52.4 \%$ were from a rural area and $47.6 \%$ from an urban area. The sex distribution was as follows: $59.5 \%$ was female and 40.5\% male. Furthermore, $45.2 \%$ were aged 12 years, $38.1 \%$ were aged 10 years, and $16.7 \%$ were aged 11 years. At the time of reassessment, the sample stratifications by age were $33.3 \%$ at 19 years, $31.0 \%$ at 20 years, $26.2 \%$ at 18 years, $4.8 \%$ at 21 years, and $4.8 \%$ at 17 years.

The dietary errors found at the initial consultation were normalized until discharge by increased consumption of vegetables and salads (initially, $47.6 \%$ consumed them daily, and at discharge all participants consumed them daily; $p<0.001$ ) and increased fruit consumption (initially, $21.4 \%$ ate 3 or more pieces, and at discharge $50 \%$ ate 3 or more pieces daily; $p<0.001$ ). The consumption of soft drinks (sugary drinks and chocolate milk) also decreased: $40.5 \%$ did not usually consume them at the initial consultation and $92.9 \%$ usually did not consume them at discharge. The same was noted for hypercaloric foods, and the habit of a healthy breakfast was acquired $(p<0.001)$. The number of daily meals increased, with $85.7 \%$ eating at least 4 meals/day at discharge $(p<0.001)$ (Table 1).

Comparison of discharge and reassessment showed that consumption of healthy breakfast $(p=1.00)$, fruits $(p=0.066)$, and soft drinks ( $p=0.1)$, as well as the number of daily meals $(p=0.422)$ was maintained. There is a tendency to increase the consumption of sweets and fast food between discharge and reassessment $(p=0.066)$, returning to the level of the initial consultation $(p=0.100)$ ( Table 1).

Regarding physical activity/inactivity, 32.5\% did not practice any sports (apart from those organized at school). Of those who practiced out-of-school sports, $15 \%$ did so at $<3 \mathrm{~h} /$ week, $20 \%$ at $3-4 \mathrm{~h} /$ week, and $32.5 \%$ over $>4 \mathrm{~h} /$ week. At discharge, all adolescents played sports and $88.1 \%$ did so for $>4 \mathrm{~h} /$ week $(p<0.001)$. This habit was lost between discharge and reassessment $(p=0.002)$, with a return to the level of the initial consultation $(p=1.00)$ (Table 1).
Intervention in Adolescents with

Overweight, Obesity, or Increased WC 
Table 3. Univariate logistic regressions for the worsening of BMI between discharge and reassessment

\begin{tabular}{|c|c|c|c|c|c|c|}
\hline & \multicolumn{2}{|l|}{$\mathrm{BMI}$} & \multirow[t]{2}{*}{$p^{*}$} & \multirow[t]{2}{*}{ OR } & \multicolumn{2}{|c|}{$95 \% \mathrm{Cl}$ for OR } \\
\hline & not worse, $n(\%)$ & worsened, $n(\%)$ & & & LL & UL \\
\hline \multicolumn{7}{|l|}{ Breakfast frequency } \\
\hline Did not worsen & $32(82.1)$ & $7(17.9)$ & \multirow{2}{*}{0.999} & \multicolumn{3}{|c|}{ Reference class } \\
\hline Worsened & $3(100.0)$ & $0(0.0)$ & & n.a. & n.a. & n.a. \\
\hline \multicolumn{7}{|l|}{ Breakfast menu } \\
\hline Did not worsen & $33(86.8)$ & $5(13.2)$ & \multirow{2}{*}{0.089} & \multicolumn{3}{|c|}{ Reference class } \\
\hline Worsened & $2(50.0)$ & $2(50.0)$ & & 6.600 & 0.751 & 58.035 \\
\hline \multicolumn{7}{|l|}{ Soup consumption } \\
\hline Did not worsen & $17(81.0)$ & $4(19.0)$ & \multirow{2}{*}{0.680} & \multicolumn{3}{|c|}{ Reference class } \\
\hline Worsened & $18(85.7)$ & $3(14.3)$ & & 0.708 & 0.138 & 3.641 \\
\hline \multicolumn{7}{|c|}{ Vegetable/salad consumption } \\
\hline Did not worsen & $19(82.6)$ & $4(17.4)$ & \multirow{2}{*}{0.890} & \multicolumn{3}{|c|}{ Reference class } \\
\hline Worsened & $16(84.2)$ & $3(15.8)$ & & 0.891 & 0.173 & 4.582 \\
\hline \multicolumn{7}{|l|}{ Meat/fish consumption } \\
\hline Did not worsen & $25(78.1)$ & $7(21.9)$ & \multirow[b]{2}{*}{0.999} & Refere & e class & \\
\hline Worsened & $10(100.0)$ & $0(0.0)$ & & n.a. & n.a. & n.a. \\
\hline Fruit consumption & & & & & & \\
\hline Did not worsen & $29(93.5)$ & $2(6.5)$ & & Refere & e class & \\
\hline Worsened & $6(54.5)$ & $5(45.5)$ & 0.009 & 12.083 & 1.880 & 77.665 \\
\hline Hypercaloric food consun & & & & & & \\
\hline Did not worsen & $22(88.0)$ & $3(12.0)$ & & Refere & e class & \\
\hline Worsened & $13(76.5)$ & $4(23.5)$ & 0.333 & 2.256 & 0.435 & 11.708 \\
\hline Soft drink consumption & & & & & & \\
\hline Did not worsen & $24(92.3)$ & $2(7.7)$ & & Refere & e class & \\
\hline Worsened & $11(68.8)$ & $5(31.3)$ & 0.063 & 5.455 & 0.912 & 32.617 \\
\hline Daily meals, $n$ & & & & & & \\
\hline Did not worsen & $26(92.9)$ & $2(7.1)$ & & Refere & e class & \\
\hline Worsened & $9(64.3)$ & $5(35.7)$ & 0.032 & 7.222 & 1.186 & 43.979 \\
\hline Out-of-school sports & & & & & & \\
\hline Did not worsen & $20(95.2)$ & $1(4.8)$ & & Refere & e class & \\
\hline Worsened & 15 (71.4) & $6(28.6)$ & 0.066 & 8.000 & 0.869 & 73.683 \\
\hline Energy expenditure & & & & & & \\
\hline Gave up & $14(73.7)$ & $5(26.3)$ & & Refere & e class & \\
\hline Maintained & $21(91.3)$ & $2(8.7)$ & 0.144 & 0.267 & 0.045 & 1.572 \\
\hline Daily walks $>30$ min & & & & & & \\
\hline Did not worsen & $12(92.3)$ & $1(7.7)$ & & Refere & e class & \\
\hline Worsened & $23(79.3)$ & $6(20.7)$ & 0.316 & 3.130 & 0.337 & 29.086 \\
\hline Sedentary daily habits $>2$ & & & & & & \\
\hline Did not worsen & $32(82.1)$ & $7(17.9)$ & & Refere & e class & \\
\hline Worsened & $3(100.0)$ & $0(0.0)$ & 0.999 & n.a. & n.a. & n.a. \\
\hline Age, years & & & & & & \\
\hline $17-18$ & $12(92.3)$ & $1(7.7)$ & 0.128 & 0.167 & 0.017 & 1.671 \\
\hline 19 & $13(92.9)$ & $1(7.1)$ & 0.111 & 0.154 & 0.015 & 1.534 \\
\hline $20-21$ & $10(66.7)$ & $5(33.3)$ & & Refere & e class & \\
\hline Gender & & & & & & \\
\hline Male & $14(82.4)$ & $3(17.6)$ & & Refere & e class & \\
\hline Female & $21(84.0)$ & $4(16.0)$ & 0.888 & 0.889 & 0.172 & 4.594 \\
\hline Area & & & & & & \\
\hline Rural & $15(75.0)$ & $5(25.0)$ & & Refere & e class & \\
\hline Urban & $20(90.9)$ & $2(9.1)$ & 0.183 & 0.300 & 0.051 & 1.763 \\
\hline Level of education & & & & & & \\
\hline Worker & $2(66.7)$ & $1(33.3)$ & & Refere & e class & \\
\hline Last year high school & $11(91.7)$ & $1(8.3)$ & 0.290 & 0.182 & 0.008 & 4.263 \\
\hline Higher education & $22(81.5)$ & $5(18.5)$ & 0.551 & 0.455 & 0.034 & 6.055 \\
\hline
\end{tabular}

n.a., not applicable; $\mathrm{Cl}$, confidence interval; OR, odds ratio; LL, lower limit; UL, upper limit; BMI, body mass index. * Wald's test $p$ value. 
Table 4. Univariate logistic regressions for the worsening of FM between discharge and reassessment

\begin{tabular}{|c|c|c|c|c|c|c|}
\hline & \multicolumn{2}{|l|}{ FM } & \multirow[t]{2}{*}{$p^{*}$} & \multirow[t]{2}{*}{ OR } & \multicolumn{2}{|c|}{$95 \% \mathrm{Cl}$ for OR } \\
\hline & not worse, $n(\%)$ & worsened, $n$ (\%) & & & LL & UL \\
\hline \multicolumn{7}{|l|}{ Breakfast frequency } \\
\hline Did not worsen & $29(74.4)$ & $10(25.6)$ & \multirow{2}{*}{0.771} & \multicolumn{3}{|c|}{ Reference class } \\
\hline Worsened & $2(66.7)$ & $1(33.3)$ & & 1.450 & 0.118 & 17.767 \\
\hline \multicolumn{7}{|l|}{ Breakfast menu } \\
\hline Did not worsen & $29(76.3)$ & $9(23.7)$ & \multirow{2}{*}{0.274} & \multicolumn{3}{|c|}{ Reference class } \\
\hline Worsened & $2(50.0)$ & $2(50.0)$ & & 3.222 & 0.395 & 26.255 \\
\hline \multicolumn{7}{|l|}{ Soup consumption } \\
\hline Did not worsen & $15(71.4)$ & $6(28.6)$ & \multirow{2}{*}{0.726} & \multicolumn{3}{|c|}{ Reference class } \\
\hline Worsened & $16(76.2)$ & $5(23.8)$ & & 0.781 & 0.197 & 3.106 \\
\hline \multicolumn{7}{|c|}{ Vegetable/salad consumption } \\
\hline Did not worsen & $17(73.9)$ & $6(26.1)$ & \multirow[b]{2}{*}{0.987} & \multicolumn{3}{|c|}{ Reference class } \\
\hline Worsened & $14(73.7)$ & $5(26.3)$ & & 1.012 & 0.254 & 4.029 \\
\hline \multicolumn{7}{|l|}{ Meat/fish consumption } \\
\hline Did not worsen & $22(68.8)$ & $10(31.3)$ & \multirow{2}{*}{0.209} & Referer & class & \\
\hline Worsened & $9(90.0)$ & $1(10.0)$ & & 0.244 & 0.027 & 2.200 \\
\hline Fruit consumption & & & & & & \\
\hline Did not worsen & $28(90.3)$ & $3(9.7)$ & & Referer & class & \\
\hline Worsened & $3(27.3)$ & $8(72.7)$ & $<0.001$ & 24.889 & 4.186 & 148.00 \\
\hline Hypercaloric food consun & & & & & & \\
\hline Did not worsen & $21(84.0)$ & $4(16.0)$ & & Referer & class & \\
\hline Worsened & $10(58.8)$ & $7(41.2)$ & 0.077 & 3.675 & 0.870 & 15.525 \\
\hline Soft drink consumption & & & & & & \\
\hline Did not worsen & $23(88.5)$ & $3(11.5)$ & & Referer & class & \\
\hline Worsened & $8(50.0)$ & $8(50.0)$ & 0.010 & 7.667 & 1.624 & 36.184 \\
\hline Daily meals, $n$ & & & & & & \\
\hline Did not worsen & $25(89.3)$ & $3(10.7)$ & & Referer & class & \\
\hline Worsened & $6(42.9)$ & $8(57.1)$ & 0.003 & 11.111 & 2.247 & 54.940 \\
\hline Out-of-school sports & & & & & & \\
\hline Did not worsen & $18(85.7)$ & $3(14.3)$ & & Referer & class & \\
\hline Worsened & $13(61.9)$ & $8(38.1)$ & 0.089 & 3.692 & 0.819 & 16.656 \\
\hline Energy expenditure & & & & & & \\
\hline Gave up & $12(63.2)$ & $7(36.8)$ & & Referer & class & \\
\hline Maintained & $19(82.6)$ & $4(17.4)$ & 0.161 & 0.361 & 0.087 & 1.501 \\
\hline Daily walks $>30$ min & & & & & & \\
\hline Did not worsen & $12(92.3)$ & $1(7.7)$ & & Referer & class & \\
\hline Worsened & $19(65.5)$ & $10(34.5)$ & 0.097 & 6.316 & 0.715 & 55.814 \\
\hline Daily sedentary habits $>2$ & & & & & & \\
\hline Did not worsen & $28(71.8)$ & $11(28.2)$ & & Referer & class & \\
\hline Worsened & $3(100.0)$ & $0(0.0)$ & 0.999 & n.a. & n.a. & n.a. \\
\hline Age, years & & & & & & \\
\hline $17-18$ & $11(84.6)$ & $2(15.4)$ & 0.163 & 0.273 & 0.044 & 1.695 \\
\hline 19 & $11(78.6)$ & $3(21.4)$ & 0.286 & 0.409 & 0.079 & 2.114 \\
\hline $20-21$ & $9(60.0)$ & $6(40.0)$ & & Referer & class & \\
\hline Gender & & & & & & \\
\hline Male & $13(76.5)$ & $4(23.5)$ & & Referer & class & \\
\hline Female & $18(72.0)$ & $7(28.0)$ & 0.747 & 1.264 & 0.305 & 5.232 \\
\hline Area & & & & & & \\
\hline Rural & $11(55.0)$ & $9(45.0)$ & & Referer & class & \\
\hline Urban & $20(90.9)$ & $2(9.1)$ & 0.015 & 0.122 & 0.022 & 0.669 \\
\hline Level of education & & & & & & \\
\hline Worker & $0(0.0)$ & $3(100.0)$ & & Referer & class & \\
\hline Last year high school & $11(91.7)$ & $1(8.3)$ & 0.999 & n.a. & n.a. & n.a. \\
\hline Higher education & $20(74.1)$ & $7(25.9)$ & 0.999 & n.a. & n.a. & n.a. \\
\hline
\end{tabular}

n.a., not applicable; $\mathrm{Cl}$, confidence interval; OR, odds ratio; LL, lower limit; UL, upper limit; FM, fat mass. * Wald's test $p$ value.

Intervention in Adolescents with

Overweight, Obesity, or Increased WC
Obes Facts 2021;14:622-632

DOI: $10.1159 / 000519270$ 
Table 5. Univariate logistic regressions for the worsening of WC between discharge and reassessment

\begin{tabular}{|c|c|c|c|c|c|c|}
\hline & \multicolumn{2}{|l|}{ WC } & \multirow[t]{2}{*}{$p^{*}$} & \multirow[t]{2}{*}{ OR } & \multicolumn{2}{|c|}{$95 \% \mathrm{Cl}$ for OR } \\
\hline & not worse, $n(\%)$ & worsened, $n(\%)$ & & & LL & UL \\
\hline \multicolumn{7}{|l|}{ Breakfast frequency } \\
\hline Did not worsen & $32(82.1)$ & $7(17.9)$ & \multirow{2}{*}{0.999} & \multicolumn{3}{|c|}{ Reference class } \\
\hline Worsened & $3(100.0)$ & $0(0.0)$ & & n.a. & n.a. & n.a. \\
\hline \multicolumn{7}{|l|}{ Breakfast menu } \\
\hline Did not worsen & $31(81.6)$ & $7(18.4)$ & \multirow{2}{*}{0.999} & \multicolumn{3}{|c|}{ Reference class } \\
\hline Worsened & $4(100.0)$ & $0(0.0)$ & & n.a. & n.a. & n.a. \\
\hline \multicolumn{7}{|l|}{ Soup consumption } \\
\hline Did not worsen & $17(81.0)$ & $4(19.0)$ & \multirow{2}{*}{0.680} & \multicolumn{3}{|c|}{ Reference class } \\
\hline Worsened & $18(85.7)$ & $3(14.3)$ & & 0.708 & 0.138 & 3.641 \\
\hline \multicolumn{7}{|c|}{ Vegetable/salad consumption } \\
\hline Did not worsen & $18(78.3)$ & $5(21.7)$ & \multirow[b]{2}{*}{0.341} & \multicolumn{3}{|c|}{ Reference class } \\
\hline Worsened & $17(89.5)$ & $2(10.5)$ & & 0.424 & 0.072 & 2.483 \\
\hline \multicolumn{7}{|l|}{ Meat/fish consumption } \\
\hline Did not worsen & $25(78.1)$ & $7(21.9)$ & \multirow{2}{*}{0.999} & Refere & class & \\
\hline Worsened & $10(100.0)$ & $0(0.0)$ & & n.a. & n.a. & n.a. \\
\hline Fruit consumption & & & & & & \\
\hline Did not worsen & $28(90.3)$ & $3(9.7)$ & & Refere & class & \\
\hline Worsened & $7(63.6)$ & $4(36.4)$ & 0.055 & 5.333 & 0.964 & 29.511 \\
\hline Hypercaloric food consun & & & & & & \\
\hline Did not worsen & $22(88.0)$ & $3(12.0)$ & & Refere & class & \\
\hline Worsened & $13(76.5)$ & $4(23.5)$ & 0.333 & 2.256 & 0.435 & 11.708 \\
\hline Soft drink consumption & & & & & & \\
\hline Did not worsen & $22(84.6)$ & $4(15.4)$ & & Refere & class & \\
\hline Worsened & $13(81.3)$ & $3(18.8)$ & 0.777 & 1.269 & 0.245 & 6.586 \\
\hline Daily meals, $n$ & & & & & & \\
\hline Did not worsen & $24(85.7)$ & $4(14.3)$ & & Refere & class & \\
\hline Worsened & $11(78.6)$ & $3(21.4)$ & 0.561 & 1.636 & 0.312 & 8.592 \\
\hline Out-of-school sports & & & & & & \\
\hline Did not worsen & $17(81.0)$ & $4(19.0)$ & & Refere & class & \\
\hline Worsened & $18(85.7)$ & $3(14.3)$ & 0.680 & 0.708 & 0.138 & 3.641 \\
\hline Energy expenditure & & & & & & \\
\hline Gave up & $17(89.5)$ & $2(10.5)$ & & Refere & class & \\
\hline Maintained & $18(78.3)$ & $5(21.7)$ & 0.341 & 2.361 & 0.403 & 13.844 \\
\hline Daily walks $>30 \mathrm{~min}$ & & & & & & \\
\hline Did not worsen & $12(92.3)$ & $1(7.7)$ & & Refere & class & \\
\hline Worsened & $23(79.3)$ & $6(20.7)$ & 0.316 & 3.130 & 0.337 & 29.086 \\
\hline Daily sedentary habits $>2$ & & & & & & \\
\hline Did not worsen & $32(82.1)$ & $7(17.9)$ & & Refere & e class & \\
\hline Worsened & $3(100.0)$ & $0(0.0)$ & 0.999 & n.a. & n.a. & n.a. \\
\hline Age, years & & & & & & \\
\hline $17-18$ & $10(76.9)$ & $3(23.1)$ & 0.506 & 1.950 & 0.272 & 13.983 \\
\hline 19 & $12(85.7)$ & $2(14.3)$ & 0.941 & 1.083 & 0.131 & 8.946 \\
\hline 20-21 & $13(86.7)$ & $2(13.3)$ & & Refere & class & \\
\hline Gender & & & & & & \\
\hline Male & $15(88.2)$ & $2(11.8)$ & & Refere & class & \\
\hline Female & $20(80.0)$ & $5(20.0)$ & 0.487 & 1.875 & 0.319 & 11.021 \\
\hline Area & & & & & & \\
\hline Rural & $16(80.0)$ & $4(20.0)$ & & Refere & class & \\
\hline Urban & $19(86.4)$ & $3(13.6)$ & 0.582 & 0.632 & 0.123 & 3.249 \\
\hline Level of education & & & & & & \\
\hline Worker & $2(66.7)$ & $1(33.3)$ & & Refere & class & \\
\hline Last year high school & $10(83.3)$ & $2(16.7)$ & 0.527 & 0.400 & 0.023 & 6.848 \\
\hline Higher education & $23(85.2)$ & $4(14.8)$ & 0.430 & 0.348 & 0.025 & 4.801 \\
\hline
\end{tabular}

n.a., not applicable; $\mathrm{Cl}$, confidence interval; $\mathrm{OR}$, odds ratio; $\mathrm{LL}$, lower limit; UL, upper limit; WC, waist circumference. ${ }^{*}$ Wald's test $p$ value. 
A similar event occurred with the habit of daily walks $(p<0.001)$, which returned to the level of the initial consultation $(p=0.72)$. Regarding sedentary habits, $78.0 \%$ of the adolescents spent $>2 \mathrm{~h} /$ day on sedentary habits at discharge and only $7.1 \%$ maintained this habit $(p<0.001)$. At reassessment, the same values and healthy habits were maintained, with no differences between discharge and reassessment $(p=1.00)$ (Table 1).

In the anthropometric assessment, $47.6 \%$ of adolescents had a normal BMI, 23.8\% were overweight, and $28.6 \%$ were obese. Moreover, $83.3 \%$ had increased FM and $4.8 \%$ had increased WC (Table 2). At discharge, all had a normal BMI $(p<0.001)$, and at reassessment, most maintained an adequate BMI. The comparison between initial consultation and reassessment showed statistically significant differences in improved BMI $(p=0.023)$. The patients maintained good BMI results between discharge and reassessment $(p=0.900)$. There was a significant improvement in WC and FM between the initial consultation and discharge $(p<0.001)$, which was maintained until reassessment ( $p=0.305$ and $p=1.00$, respectively) (Table 2).

We performed univariable logistic regressions for worsening BMI, FM, and WC between discharge and reassessment to identify possible causes for the association between changes in eating habits and physical activity/ inactivity or between gender, environment, and age (Tables 3-5). There appears to be an association between decreased fruit consumption and increased BMI because $45.5 \%$ of adolescents with increased BMI had decreased fruit consumption. The decrease in the number of daily meals, increase in the consumption of soft drinks, and increase in FM were all associated with a decrease in fruit consumption, whereby $72.7 \%$ of adolescents with increased FM between discharge and reassessment had a decrease in both daily fruit consumption $(p<0.001)$ and number of meals $(p=0.003)$. Furthermore, there was a slight association between increased consumption of soft drinks and a decrease in sports activities. The increase in WC was also associated with decreased fruit consumption $(p=0.055)$ (Table 5).

\section{Discussion}

Initially, all adolescents in the sample had altered BMI values: $23.8 \%$ were overweight and $28.6 \%$ were obese. Moreover, $83.3 \%$ had increased FM, and $95.2 \%$ had increased WC. This is related to the increased risk of developing various diseases $[25,26]$. Unhealthy eating habits

Intervention in Adolescents with

Overweight, Obesity, or Increased WC were identified, including excess consumption of hypercaloric foods/drinks, especially at breakfast (47.6\%), and excess consumption of soft/sugary drinks (33.4\% consumed daily/or $>4 \times$ week), with the daily consumption of vegetables/salads being low at $47.6 \%$ and most adolescents consuming only 1 piece of fruit daily.

Studies show that hypercaloric drinks/foods should be consumed in moderation because they lead to obesity and increased FM [27]. For example, in the UK, $>70 \%$ of adolescents regularly consume sugary/carbonated drinks [28].

In our study, $78 \%$ of adolescents had sedentary habits for $>2 \mathrm{~h} /$ day, which is consistent with the Bray et al. [25] study. A sedentary lifestyle is associated with a higher prevalence of overweight/obesity and increased consumption of hypercaloric foods/drinks $[29,30]$. The National Heart, Lung, and Blood Institute Growth and Health Study recorded a decrease in physical exercise throughout adolescence, showing an association with increased BMI [31]. At the initiation of the study, approximately $32.5 \%$ of the adolescents practiced sports for $>4 \mathrm{~h} /$ week and $27.5 \%$ took daily walks ( $>30 \mathrm{~min}$ ). During quarterly consultations, exercise, decreased sedentarism, and a healthier diet were promoted because we required all adolescents to practice out-of-school sports for a minimum of $4 \mathrm{~h} /$ week to decrease their sedentary activities to $<2 \mathrm{~h} /$ day.

After 3 years of follow-up, practically all adolescents had healthier habits, and their BMI, FM, and WC were normalized. Similar to previous studies, our study highlights the importance of exercise during adolescence, especially for weight control and BMI [32-36].

Moreover, we believe that the decrease in sedentary lifestyle helped normalize the altered parameters, similar to the finding of Kaur et al. [36] who concluded that adolescents who watch television for $>2 \mathrm{~h} /$ day have a higher risk of increased BMI than adolescents who watch television for $<2 \mathrm{~h} /$ day. In our study, the healthy habits observed at discharge regarding sedentary lifestyle remained high until reassessment, as did most values of BMI, WC, and FM. The success achieved was also owing to the implementation of healthier habits, especially consumption of healthy breakfast daily, $>4$ meals/ day, minimum 3 pieces of fruit daily, and vegetables/ salads daily; lower consumption of hypercaloric foods/ drinks; and reduction in meal portions. Family involvement was a decisive factor. Other studies have showed similar results $[37,38]$.

When comparing discharge and reassessment, there was a slight increase in FM in some adolescents and BMI 
and WC in others, but this was not statistically significant. We identified a direct relationship between increased FM and decreased fruit consumption. The transition to adulthood mandated these young individuals to leave their homes, making them responsible for their own meals [38]. The lack of time and lack of sports facilities led them to neglect exercise, diet, and number of meals, as analyzed in our study [39]. Steinbeck et al. [40] demonstrated that strategies to combat obesity depend more on patients' participation in adolescence than that in childhood and must recognize the patients' progressive autonomy. Promoting the association between a healthy diet, exercise, and low sedentary lifestyle from an early age and motivating the adolescent to maintain these habits during adulthood would be ideal yielding the best results [40-42]. The involvement of schools, the government, and family is essential for this success [17, 19-21].

As stated previously, we found results similar to ours in other studies. Some of them demonstrated similar conclusions regarding a specific topic approached by us (like the relation between the number of hours watching television and obesity, or between the consumption of fruits and vegetables or salads and overweight or even the effect that the family has in preventing overweight and obesity during adolescence), but we could not find a study that incorporated all these aspects (diet, exercise, level of sedentarism, and family's participation) in 1 single structured medical intervention like ours, in that regard, our study is innovative.

The limitations of our study are the small sample size and limited number of locations in which the study was performed. Forty-two participants is not a high number; perhaps, better results could have been achieved if the sample size was 100 adolescents or more. Equally, the higher the number of locations in which the study is performed, the more randomized and scientifically credible it is. New studies are warranted with a higher number of participants and at more locations of the country, including in both rural and urban areas, to obtain a more diverse data group to confirm the findings of this study.

\section{Conclusions}

This study demonstrated the effectiveness of a structured medical intervention in adolescents with obesity/ overweight or increased WC. It is proven that when primary prevention fails, it is possible to not only correct obesity/overweight and increased FM and WC but also prolong the results of this intervention over time, at least up to 5 years, with predictable significant gains in adolescents' future health.

The success of our intervention is owing to the fact that it was aimed at adolescents and families and was based on the implementation of healthier eating habits, increased physical exercise ( $>4 \mathrm{~h} /$ week), daily walks ( $>30 \mathrm{~min}$ ), and decreased sedentary habits. Maintaining the healthy lifestyle habits learned during the medical intervention contributed to the maintenance of normal BMI and adiposity.

Implementing healthy eating habits as well as exercise and decreasing physical inactivity are fundamental for improving BMI, FM, and WC in adolescents. Our study is innovative in the field, demonstrating that motivation and involvement of family and government are essential to ensure the success of these measures. Over the years, a tendency to abandon some initial healthy habits achieved, particularly with respect to food, develops. However, other habits remain, for example, most adolescents still spend $<2 \mathrm{~h}$ /day in sedentary habits, maintain a healthy breakfast, eat $>4$ meals/day, and not consume hypercaloric food/drinks daily. Simultaneously, the parameters of BMI, FM, and WC remain mostly normal. Therefore, the need, feasibility, and effectiveness of the short- and medium-term structured medical intervention with family involvement in obese/overweight adolescents are therefore proved.

\section{Statement of Ethics}

This is a prospective cohort study that was approved by the Ethics Committee of the Pediatric Hospital of Coimbra (1022) and the Faculty of Medicine of the University of Coimbra (G/295). All participants and guardians received written explanation regarding the study and its objectives, and informed free consent was obtained. The final work observes all appropriate criteria for integrity, ethics, and data interpretation.

\section{Conflict of Interest Statement}

The authors have no conflicts of interest to declare.

\section{Funding Sources}

This research did not receive any specific grants from funding agencies in the public, commercial, or not-for-profit sectors. 


\section{Author Contributions}

Luísa Macieira contributed to conception, design, and execution of this study; analysis and interpretation of the results; and writing of the article. Jorge Saraiva contributed to the conception, writing, and review of the study and approval of the final version. Lélita Santos made an important contribution to the design of the study and result interpretation; critical review; and approval of the final version to be published.

\section{Data Availability Statement}

Due to ethical concerns, as this cohort is easily identifiable because it respects only 1 center and also it is part of another larger project, data cannot be publicly available. In case the readers want to access the data, they can contact the authors.

\section{References}

1 Mäkelä J, Lagström H, Pitkänen N, Kuulasmaa T, Kaljonen A, Laakso M, et al. Genetic risk clustering increases children's body weight at 2 years of age: the STEPS study. Ped Obes. 2016 Dec;11:459-67.

2 World Health Organization [Internet]. Obesity. Preventing and managing the global epidemic: report of a W.H.O consultation on obesity. Geneva; 1997. Available from: https: //www.who.int/nutrition/publications/obesity/WHO_TRS_894/en/.

3 Speiser PW, Rudolf MC, Anhalt H, CamachoHubner C, Chiarelli F, Eliakim A, et al. Childhood obesity. J Clin Endocrinol Metab. 2005 Mar;90(3):1871-87.

4 Lissau I, Overpeck MD, Ruan WJ, Due P, Holstein BE, Hediger ML, et al. Body mass index and overweight in adolescents in 13 European countries, Israel, and the United States. Arch Pediatr Adolesc Med. 2004 Jan;158:27-33.

5 Ebbeling CB, Pawlak DB, Ludwig DS. Childhood obesity: public-health crisis, common sense cure. Lancet. 2002 Aug;360:473-82.

6 González-Munieza P, Mártinez-González MA, Hu FB, Després JP, Matsuzawa Y, Loos RJF, et al. Obesity. Nat Rev Dis Primers. 2017 Jun;3:17034.

7 Gong CD, Wu QL, Chen Z, Zhang D, Zhao ZY, Peng YM. Glycolipid metabolic status of overweight/obese adolescents aged 9-15-year-old and the BMI-SDS/BMI cut-off value of predicting dyslipidemia in boys, Shanghai, China: a cross-sectional study. Lipids Health Dis. 2013 Aug;12:1-9.

8 Padez C, Mourão I, Moreira P, Rosado V. Prevalence and risk factors for overweight and obesity in Portuguese children. Acta Paediatr. 2005 Nov;94(11):1550-7.

9 Proctor MH, Moore LL, Gao D, Cupples LA, Bradlee ML, Hood MY, et al. Television viewing and change in body fat from preschool to early adolescence: the Framingham children's study. Int J Obes Relat Metab Disord. 2003 Jul;27:827-33.

10 World Health Organization [Internet]. Childhood overweight and obesity; 2019 Mar 5. Available from: http://www.who.int/dietphysicalactivity/childhood/en/.
11 Pascual-Gamarra JM, Salazar-Tortosa D Martinez-Tellez B, Labayen I, RupérezCensi AIL, Censi L, et al. Association between UCP1, UCP2, and UCP3 gene polymorphisms with markers of adiposity in European adolescents: the HELENA study. Pediatr Obes. 2019 Jun; 14:e12504-6.

12 Lobstein T, Jackson-Leach R, Moodie ML, Hall KD, Gortmaker SL, Swinburn BA, et al. Child and adolescent obesity: part of a bigger picture. Lancet. 2015;385:2510-20.

13 Kelley G, Kelley KS. Effects of exercise in the treatment of overweight and obese children and adolescents: a systematic review of metaanalyses. J Obes. 2013;2013:783103.

14 Kuźbicka K, Rachoń D. Bad eating habits as the main cause of obesity among children. Ped Endocrinol Diabetes Metab. 2013;19(3): 106-10.

15 Weker H. Simple obesity in children: a study on the role of nutritional factors. Med Wieku Rozwoj. 2006 Jan-Mar;10(1):3-191.

16 Bellew W, Bauman A, Kite J, Foley B, Reece L, Thomas M, et al. Obesity prevention in children and young people: what policy actions are needed? Public Health Res Pract. 2019 Mar;29(1):1-6.

17 Wang Y, Cai L, Wu Y, Wilson RF, Weston C, Fawole $\mathrm{O}$, et al. What childhood obesity prevention programs work? A systematic review and meta-analysis. Obes Rev. 2015 Jul;16: 547-65.

18 Yeager LJ, Karp SM, Leming-Lee T. Barriers to the implementation of pediatric overweight and obesity guidelines in a schoolbased health center. Nurs Clin North Am. 2019 Mar;54(1):159-68.

19 Wilfley DE, Stein RI, Saelens BE, Mockus DS, Matt GE, Hayden-Wade HA, et al. Efficacy of maintenance treatment approaches for childhood overweight: a randomized controlled trial. JAMA. 2007 Oct;298(14):1661-73.

20 Gerards SM, Sleddens EF, Dagnelie PC, De Vries NK, Kremers SP. Interventions addressing general parenting to prevent or treat childhood obesity. Int J Pediatr Obes. 2011 Jun;6:e28-45.

21 Golden NH, Schneider M, Wood C. Preventing obesity and eating disorders in adolescents. Pediatrics. 2016 Sept;138(3):10.
22 Macieira L, Saraiva J, Santos L. Overweight and obesity and their associated factors among early adolescence school children in urban and rural Portugal. BMC Nutrition. 2017 Feb;3:17.

23 Portugal, Ministério da Saúde, Direção Geral da Saúde. Saúde infantil e juvenil: programatipo de atuação. Lisbon: Direção Geral da Saúde; 2013.

24 Frisancho AR. Anthropometric standards for the assessment of growth and nutritional status. 1st ed. Ann Arbor: The University of Michigan Press; 1990.

25 Bray GA, Clearfield MB, Fintel DJ, Nelinson DS. Overweight and obesity: the pathogenesis of cardiometabolic risk. Clin Cornerstone. 2009;9(4):30-2.

26 Guillaume M, Lapidus L, Beckers F, Lambert A, Bjömtorpet P. Cardiovascular risk factors in children from the Belgian province of Luxembourg: the Belgian Luxembourg child study. Am J Epidemiol. 1996;144(9):867-80.

27 Ludwig DS, Peterson KE, Gortmaker SL. Relation between consumption of sugar-sweetened drinks and childhood obesity: a prospective observational analysis. Lancet. 2001 Feb; 357:505-8.

28 Hamack L, Stang J, Story M. Soft drink consumption among US children and adolescents: nutritional consequences. J Am Diet Association. 1999 Apr;99(4):436-41.

29 Faith MS, Berman N, Heo M, Pietrobelli A, Gallagher D, Epstein LH, et al. Effects of contingent television on physical activity and television viewing in obese children. Pediatrics. 2001 May;107(05):1043-8.

30 Pearson N, Biddle SJ. Sedentary behavior and dietary intake in children, adolescents, and adults: a systematic review. Am J Prev Med. 2011 Aug;41(2):178-88.

31 Kymm SY, Obarzanek E. Childhood obesity: a new pandemic of the new millennium. Ped. 2002 Nov;110(5):1003-7.

32 Gunner KB, Atkinson PM, Nichols J, Eissa MA. Health promotion strategies to encourage physical activity in infants, toddlers, and preschoolers. J Pediatr Health Care. 2005 Jul; 19(4):253-8.
Intervention in Adolescents with

Overweight, Obesity, or Increased WC
Obes Facts 2021;14:622-632 
33 Flynn MA, McNeil DA, Maloff B, Mutasingwa D, Wu M, Ford C, et al. Reducing obesity and related chronic disease risk in children and youth: a synthesis of evidence with "best practice" recommendations. Obes Rev. 2006 Feb;7(Suppl 1):7-66.

34 Hallal PC, Victora CG, Azevedo MR, Wells JC. Adolescent physical activity and health: a systematic review. Sports Med. 2006;36(12): 1019-30.

35 Atlantis E, Barnes EH, Singh MA. Efficacy of exercise for treating overweight in children and adolescents: a systematic review. Int $\mathrm{J}$ Obes. 2006 Jul;30(7):1027-40.
36 Kaur H, Choi WS, Mayo MS, Harris KJ. Duration of television watching is associated with increased body mass index. J Pediatr. 2003 Oct;143(4):506-11.

37 Birch LL, Savage JS, Fischer JO. Right sizing prevention: food portion size effects on children's eating and weight. Appetite. 2015 May; 88:11-6.

38 World Health Organization. Consideration of the evidence on childhood obesity for the commission on ending childhood obesity: report of the ad hoc working group on science and evidence for ending childhood obesity. Geneva: World Health Organization; 2016.
39 Mota J, Fidalgo F, Silva R, Ribeiro JC, Santos $\mathrm{R}$, Carvalho J, et al. Relationships between physical activity, obesity and meal frequency in adolescents. Ann Hum Biol. 2008 Jan; 35(1):1-10.

40 Steinbeck KS, Lister NB, Gow ML, Baur LA. Treatment of adolescent obesity. Nat Rev Endocrinol. 2018 Jun;14(6):331-44.

41 Reybrouck T, Vinckx J, Van den Berghe G, Vanderschueren-Lodeweyckx M. Exercise therapy and hypocaloric diet in the treatment of obese children and adolescents. Acta Paediatr Scand. 1990 Jan;79(1):84-9.

42 Vissers D, Hens W, Hansen D, Taeymans J. The Effect of diet or exercise on visceral adipose tissue in overweight youth. Med Sci Sports Exerc. 2016 Jul;48(7):1415-24. 